\title{
Determination of globotriaosylceramide in plasma and urine by mass spectrometry
}

\author{
Ralf Krüger $^{1, *}$, Kai Bruns ${ }^{1, a}$, Silke Grünhage ${ }^{1}$, \\ Heidi Rossmann', Jörg Reinke², Michael Beck ${ }^{2}$ \\ and Karl J. Lackner ${ }^{1}$ \\ ${ }^{1}$ Institute of Clinical Chemistry and Laboratory Medicine, \\ Medical Center of the Johannes Gutenberg University, \\ Mainz, Germany \\ ${ }^{2}$ Department of Pediatrics/Villa Metabolica, Medical \\ Center of the Johannes Gutenberg University, Mainz, \\ Germany
}

\begin{abstract}
Background: Fabry disease is an X-chromosomally inherited lysosomal storage disorder leading to accumulation of glycosphingolipids, mainly globotriaosylceramide (ceramide-trihexoside, Gb3). Concentrations of Gb3 in plasma and urine have been used to diagnose Fabry disease and to monitor enzyme replacement therapy with recombinant $\alpha$ galactosidase.

Methods: Gb3 was purified from plasma or urine by combined liquid extraction/protein precipitation and solid-phase extraction, and was detected by flow-injection analysis electrospray mass spectrometry (MS) using multi-reaction-monitoring. Calibration was performed via standard addition using $\mathrm{C} 17-\mathrm{Gb} 3$ as internal standard. The most abundant isoforms were monitored for calculation of total Gb3.

Results: A MS-based assay for quantification of Gb3 in plasma and urine was established and validated. Intra- and interassay coefficient of variation $(\mathrm{CV})$ of the method were $\leq 12 \%$. However, at low concentrations the CV was $16 \%$. The linear range covers roughly two orders of magnitude, down to $0.54 \mathrm{mg} / \mathrm{L}$ in plasma and $0.07 \mathrm{mg} / \mathrm{L}$ in urine. Careful adjustment of tuning parameters was necessary to obtain identical isoform intensities and quantitative results on different mass spectrometers. Gb3 concentrations in healthy controls were $<4 \mathrm{mg} / \mathrm{L}$ in EDTA-plasma and $<10 \mu \mathrm{g} / \mathrm{mmol}$ creatinine in urine. Significantly increased Gb3 concentrations were found in plasma and urine from male and female patients with Fabry disease.
\end{abstract}

\footnotetext{
apresent address: Sanofi-Aventis Deutschland GmbH, Industriepark Hoechst, Frankfurt, Germany.

*Corresponding author: Dr. Ralf Krüger, Institute of Clinical Chemistry and Laboratory Medicine, Medical Center of the Johannes Gutenberg University, Langenbeckstr. 1, 55131 Mainz, Germany

E-mail: krueger@zentrallabor.klinik.uni-mainz.de Received July 10, 2009; accepted October 7, 2009; previously published online December 4, 2009
}

Conclusions: An improved MS protocol for Gb3 quantification has been developed, validated, and shown to be suitable for diagnosis and monitoring of Fabry patients.

Clin Chem Lab Med 2010;48:189-98.

Keywords: Fabry disease; Gb3 (globotriaosylceramide); glycosphingolipid; liquid chromatography-tandem mass spectrometry; lysosomal storage disorder.

\section{Introduction}

Globotriaosylceramide (Gb3), a trisaccharide linked sphingolipid (see Figure 1 for structure details), is the most abundant and common accumulation product in patients with Fabry disease, an X-chromosomally inherited lysosomal storage disorder (1-3). The genetic defect affects the enzyme $\alpha$-galactosidase which is necessary to cleave a terminal, nonreducing $\alpha$-D-galactose from a glycan moiety $(4,5)$. As a consequence, this glycan degradation pathway is blocked and corresponding glycans, including Gb3, accumulate in tissues primarily in the central nervous system, skin, kidney and muscles (including heart). Typical signs are neuropathic pain, fatigue and depression, angiokeratomas, hypohydrosis, gastrointestinal disorders, eye anomalies (cornea verticilata, Fabry-cataracts), hearing impairment, chronic infection, late puberty onset, and altered or delayed skeletal growth $(6,7)$. Progression of the disease in adolescent and adult patients may compromise brain, kidney and heart function, leading to high morbidity and mortality due to renal failure and ischemic events, such as stroke and myocardial infarction (8-11).

Gb3 concentrations in plasma and urine of patients with Fabry disease were found to be increased (12), albeit with considerable individual variation (11). Quantification of Gb3 supports initial diagnosis and has been applied for monitoring the progression of Fabry disease (13). Data suggest that monitoring Gb3 may be of particular significance in cases of enzyme replacement therapy (ERT) (14).

Amphiphilic glycolipids can be extracted from tissue, blood or urine by mixtures of organic solvents, such as acetone, chloroform and methanol. Early assays for glycosphingolipid analysis were based on thin layer chromatography (15-18), gas chromatography $(15,17)$ or HPLC using reversed phase (19) or normal phase (20-23) chromatography. A method using sequential HPLC and high performance thin layer chromatography was also successfully applied (24). All these methods are usually time consuming, and require deacylation and/or derivatization steps. An immu- 


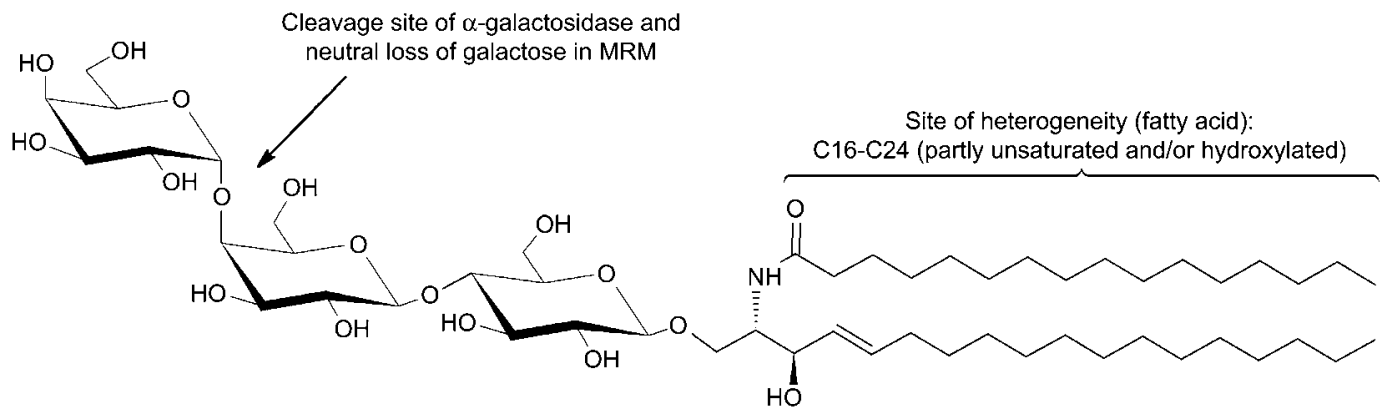

Figure 1 Structure of the glycolipid Gb3.

$\mathrm{Gb3}$ belongs to the group of neutral glycosphingoplipids and consists of a trisaccharide linked to a ceramide moiety ( $\mathrm{N}$-acylsphingosine). The trisaccharide is composed of $1-4$ linked $\beta$-D-glucose, $\beta$-D-galactose and $\alpha$-D-galactose $(\operatorname{Gal}(\alpha 1 \rightarrow 4) \mathrm{Gal}(\beta 1 \rightarrow 4) \mathrm{Glc}(\beta 1 \rightarrow 1) \mathrm{ceramide})$. The fatty acid component linked via the amide bond exhibits a high degree of heterogeneity, whereas the sphingosine alkyl chain is almost homogenous (mainly C18:1). The arrow marks the bond which is cleaved by the enzyme $\alpha$-galactosidase, lacking in Fabry patients, where also the specific fragmentation for mass spectrometric detection occurs (loss of one galactose moiety, $-162 \mathrm{~m} / \mathrm{z}$ ).

noassay (ELISA) was developed in 1999 (25). However, with the advent of mass spectrometry (MS), faster and more specific methods have been developed. Detection of Gb3 by MS has been achieved by fast-atom-bombardement (FAB) (26), and matrix-assisted laser desorption-ionization (MALDI) has been used for detection and relative quantification of $\mathrm{Gb} 3$ and other sphingolipids for disease classification (27). Tandem MS methods for absolute quantification usually rely on specific fragmentation using electrospray ionization combined with multi-reaction-monitoring (MRM). Gb3 can be monitored by a specific loss of one galactose residue $(-162 \mathrm{~m} / \mathrm{z})$ from the sodiated precursor ion. Suitable methods which use protein precipitation and liquid or solid phase extraction (SPE) in combination with flow-injection analysis-MS (FIA-MS) have been described (28-31). Due to the hydrophobic nature of $\mathrm{Gb3}$, reversed phase chromatography in combination with MS is challenging with respect to memory effects and tailing. One solution is to use very short columns with low hydrophobicity. Nelson et al. and Mills et al. described a liquid chromatography-tandem mass spectrometry (LC-MS) method that used two short C8 guard columns and a tertiary gradient to ensure complete lipid washout (32-35). Delobel et al. performed structural analysis of Gb3 species using a porous graphitic column and atmospheric pressure photoionization (APPI) (36).

However, for robust quantitative routine assays, it can be advantageous to omit the chromatographic step and to rely instead on extraction and purification steps during sample preparation. Based on earlier approaches, we describe an improved methodology using combined liquid extraction/ protein precipitation, normal phase SPE and FIA-MS-MRM, and its application to clinical monitoring of Gb3 concentrations in plasma and urine of patients with Fabry disease.

\section{Materials and methods}

\section{Patients}

Blood and urine samples from healthy volunteers and Fabry patients were collected after obtaining informed consent. Patients were included in Fabry Outcome Survey (FOS), which was approved by the Ethics Committee/Institution Review Board of all participating centers. The age (years) distribution was as follows [shown as median (mean) and range] controls plasma $(\mathrm{n}=50), 27.5$ (30.4), 19-54; male Fabry plasma $(\mathrm{n}=87), 29$ (32.0), 8-68; female Fabry plasma $(n=122), 36$ (36.1), 6-69; controls urine $(n=89), 38(40.1), 19-68$; male Fabry urine $(\mathrm{n}=71), 29$ (31.0), 8-56; female Fabry urine $(\mathrm{n}=117), 36(36.1), 8-70$.

\section{Chemicals and standards}

Chloroform p.a. (Roth, Karlsruhe, Germany), spectroscopy grade acetone, ethyl acetate p.a., HPLC-grade methanol (Merck, Darmstadt, Germany) and HPLC-grade water (Mallinckrodt-Baker, Deventer, Netherlands) were used as solvents. The porcine Gb3 calibrator and the internal standard (ISTD) heptadecanoyl-Gb3 (C17CTH) were obtained from Matreya LLC (Pleasant Gap, PA, USA) and dissolved in chloroform:methanol $(2: 1 / \mathrm{v}: \mathrm{v})$. The final concentration of the calibrator stock solution was $40 \mathrm{mg} / \mathrm{L}$, and the ISTD was dissolved and diluted to a final working concentration of $0.5 \mathrm{mg} / \mathrm{L}$. Standard addition was used for both plasma and urine samples.

\section{Sample preparation EDTA-plasma}

For each sample, standard and control, one vial containing $1200 \mu \mathrm{L}$ acetone:methanol (1:1/v:v) spiked with $20 \mu \mathrm{L}$ ISTD solution was prepared. Seven standard concentrations were prepared in duplicate by spiking with different volumes of Gb3 standard stock solution $(0-48 \mu \mathrm{L})$ to produce plasma concentrations of $0.0,1.2,2.4,4.8$, 9.6, 14.4 and $19.2 \mathrm{mg} / \mathrm{L} .100 \mu \mathrm{L}$ of EDTA plasma was added to each vial. Pooled EDTA plasma was used in case of standards and controls. Controls were prepared at two concentrations in duplicate. Samples, standards and controls were processed identically as described below. The vials were sonicated for $5 \mathrm{~min}$, vortexed and centrifuged for $10 \mathrm{~min}$ at $2800 \mathrm{~g}$ per min. The supernatant was transferred into a new vial and dried in a vacuum centrifuge. Dry samples may be stored at $-20^{\circ} \mathrm{C}$ for later processing.

\section{Sample preparation urine}

For each sample, standard and control, one vial containing $1200 \mu \mathrm{L}$ chloroform:methanol (2:1/v:v) spiked with $20 \mu \mathrm{L}$ ISTD solution was prepared. Seven standard concentrations were prepared in dupli- 
cate by spiking with different volumes of Gb3 standard stock solution $(0-36 \mu \mathrm{L})$ to produce urine concentrations of $0.0,0.3,0.6,1.2$, 2.4, 4.8 and $7.2 \mathrm{mg} / \mathrm{L} .200 \mu \mathrm{L}$ urine from a $24-\mathrm{h}$ collection was added to each vial. Pooled urine samples were used for standards and controls. Controls were prepared in duplicate at two concentrations. All other steps were identical to those described for plasma.

\section{Solid-phase-extraction}

Normal phase SPE was identical for plasma and urine samples. Silica SPE columns (Bond Elut Straight Barrel Cartridges, 100 $\mathrm{mg} / 1 \mathrm{~mL}$, Varian, Palo Alto, CA, USA), were washed and equilibrated 3 times with $1 \mathrm{~mL}$ of methanol and 3 times with $1 \mathrm{~mL}$ of chloroform. Gb3 pellets were redissolved in $1 \mathrm{~mL}$ of chloroform with subsequent sonication for $5 \mathrm{~min}$, thorough vortexing and centrifugation at $13,000 \mathrm{~g}$ for $10 \mathrm{~min}$. The redissolved samples were applied onto the SPE-columns. The columns were washed 3 times with $1 \mathrm{~mL}$ of chloroform and 3 times with $1 \mathrm{~mL}$ of ethyl acetate. Gb3 was eluted with $3 \mathrm{~mL}$ of acetone:methanol $(8: 2 / \mathrm{v}: \mathrm{v})$ and dried in a vacuum centrifuge.

\section{Mass spectrometry}

Quantification of Gb3 was performed using electrospray MS with a triple-quadrupole instrument (TSQ Quantum Discovery Max, Thermo Scientific, Waltham, MA, USA). Sample introduction was performed with a standard HPLC pump and autosampler (Surveyor, Thermo Scientific). For comparison of potential instrument effects, an ion-trap mass spectrometer (LCQ Deca with Surveyor HPLC, Thermo Scientific, Waltham, MA, USA) and another triple quadrupole mass spectrometer (Quattro Premier XE with Acquity UPLC, Waters, Milford, MA, USA) were also used.

Samples were redissolved in $100 \mu \mathrm{L}$ of methanol with subsequent sonication for $5 \mathrm{~min}$, thorough vortexing and centrifugation at $13,000 \mathrm{~g}$ for $10 \mathrm{~min}$. The supernatant was transferred into glass microvials. Samples were introduced into the mass spectrometer by FIA $(15 \mu \mathrm{L})$ using pure methanol at a flow rate of $150 \mu \mathrm{L} / \mathrm{min}$. Two short blank runs $(25 \mu \mathrm{L}$ methanol, 1.5 min run time, $200 \mu \mathrm{L} / \mathrm{min}$ ) were injected between samples for washout. Gb3 was monitored by MRM using the specific loss of one galactose unit $(-162 \mathrm{~m} / \mathrm{z})$ from the sodium adducts as a marker of fragmentation. Since Gb3 is not homogeneous with respect to the fatty acid composition, transitions for the most abundant isoforms were selected (length, saturation degree and oxidation status of the fatty acid moiety are given in parentheses): m/z 1046.7 (C16:0), m/z 1060.7 (C17:0, ISTD), m/z 1130.8 (C22:0), m/z 1146.9 (C22:0-OH), m/z 1156.9 (C24:0), m/z 1158.9 (C24:1), m/z 1174.9 (C24:0-OH).

The optimized instrument conditions were as follows:

TSQ Quantum: spray voltage $3.0 \mathrm{kV}$, sheath gas $20 \mathrm{AU}$, auxiliary gas $4 \mathrm{AU}$, capillary temperature $380^{\circ} \mathrm{C}$, source CID $7 \mathrm{~V}$, tube lens offset $230 \mathrm{~V}$, collision energy $62 \mathrm{eV}$.

Quattro Premier: spray voltage $3.7 \mathrm{kV}$, desolvation gas $500 \mathrm{~L} / \mathrm{h}$, cone gas $25 \mathrm{~L} / \mathrm{h}$, desolvation temperature $200^{\circ} \mathrm{C}$, source temperature $60^{\circ} \mathrm{C}$, cone voltage $110 \mathrm{~V}$, collision energy $68 \mathrm{eV}$.

LCQ Deca: spray voltage $3.0 \mathrm{kV}$, sheath gas $9 \mathrm{AU}$, auxiliary gas 0 $\mathrm{AU}$, capillary temperature $240^{\circ} \mathrm{C}$, source CID $100 \mathrm{~V}$, capillary voltage $45 \mathrm{~V}$, tube lens offset $35 \mathrm{~V}$, collision energy $46 \%$.

\section{Data processing}

Peak integration was checked and corrected where necessary using either Xcalibur (Thermo Scientific) or MassLynx software (Waters). Data were exported to Excel. Quantification of total Gb3 was achieved by summing up the intensities of the Gb3 isoforms and dividing by the intensity of the ISTD. Since no Gb3-free plasma is available, standard addition was applied for calibration. Gb3 concentrations in urine were normalized to urinary creatinine concentration.

\section{Results}

\section{Method evaluation}

The method was evaluated according to quality system procedures prior to approval for routine analysis. Calibration was performed via standard addition of porcine Gb3 (see Figure 2). Calibration curves (7 concentrations) were linear, with regression coefficients $\left(\mathrm{r}^{2}\right)>0.99$ for urine, and 0.98 for plasma. The lower value for plasma was due to the higher background of plasma pools used for spiking. The average of the correlation coefficients obtained between October 17, 2005 and December 31, 2007 was $r^{2}=(0.9929 \pm 0.0099)$ for urine and $r^{2}=(0.9871 \pm 0.0252)$ for plasma.

Intra- and inter-assay imprecision were measured at two concentrations, representative of Fabry patients and healthy controls (see Table 1). Pooled samples were used for this
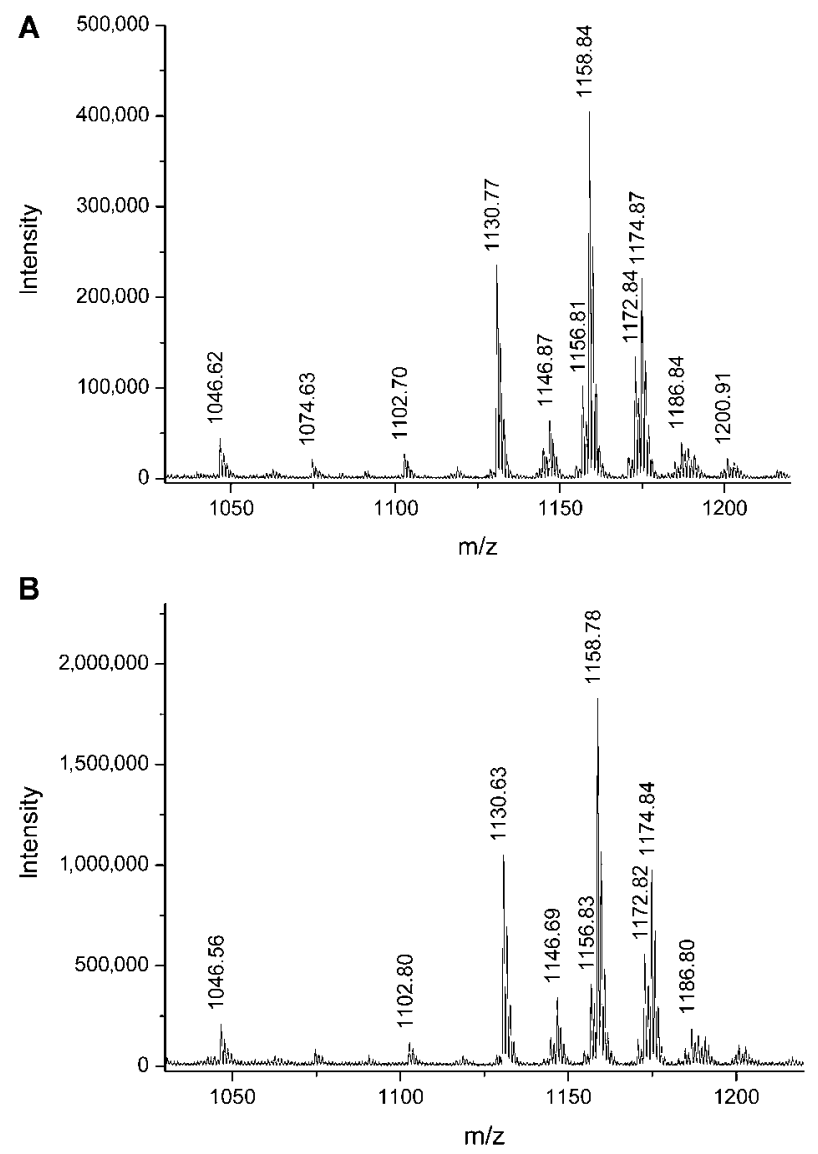

Figure 2 Mass spectrum of porcine Gb3 standard obtained using two different triple quadrupole mass spectrometers.

(A) TSQ Quantum Discovery Max (Thermo Electron); (B) Quattro Premier XE (Waters). 
purpose. Intra-assay imprecision was determined by analyzing individually prepared sample aliquots $(n=10-11)$ in one batch. Inter-assay imprecision was obtained by analyzing aliquots $(n=23-29)$ in different runs on different days. Coefficient of variation (CV) ranged from $4 \%$ to $12 \%$, with the exception of $16 \%$ for urine with low concentrations. However, inter-assay imprecision in plasma and urine was comparable at higher Gb3 concentrations.

Linearity was assessed by serial dilution of Gb3 samples. The results are expressed as recovery (deviation from the mean), together with the correlation coefficient and the assessed linear range (see Table 2). In the concentration range tested, recoveries \pm standard deviation (SD) were $100 \% \pm 4 \%$ for plasma and $100 \% \pm 2 \%$ for urine, with a minimum and maximum recovery of $92 \%$ and $108 \%$, respectively. Regression coefficients were $>0.995$ for plasma and $>0.999$ for urine. The method proved to be linear down to $0.54 \mathrm{mg} / \mathrm{L}$ for EDTA plasma and down to $0.07 \mathrm{mg} / \mathrm{L}$ for urine (two dilution series). The signal-to-noise ratio (S/N) upon repeated analysis of patient pools was used to calculate analytical sensitivity. The limit of detection ( $\mathrm{S} / \mathrm{N} 3: 1)$ was $0.02 \mathrm{mg} / \mathrm{L}$ for plasma and $0.01 \mathrm{mg} / \mathrm{L}$ for urine.

Aliquots from Fabry patient pools (two concentrations) were frozen at $-20^{\circ} \mathrm{C}$ and used as quality control samples.
Frozen plasma samples were stable for at least 1 year, whereas Gb3 in urine samples showed a decrease in the low concentration range of up to $20 \%$ after 1 year (compare stability values in Table 3 ).

\section{Instrument comparison}

Initial experiments for method development have been performed with a quadrupole ion trap (LCQ Deca, Thermo Electron) using selective reaction monitoring. After subsequent evaluation and method approval of a MRM method with a triple quadrupole (TSQ Quantum, Thermo Electron), a set of 89 patient samples was used to perform an instrument comparison (Figure 3A). Correlation between the results was high $\left(r^{2}>0.96\right)$, but the ion trap results were systematically lower. Consequently, the routine assay was performed exclusively with the triple quadrupole, an instrument type which is known for its excellent quantification performance.

With the growing number of samples from two multicenter studies [FOS (37-39), and Stroke in Young Fabry Patients (SIFAP) (10)], the question of a method transfer to another instrument became apparent. Thus, we started to evaluate the method using another triple quadrupole MS (Quattro Premier $\mathrm{XE}$, Waters). To our surprise, distinct deviations were

Table 1 Imprecision results for Gb3 quantification.

\begin{tabular}{|c|c|c|c|c|c|c|}
\hline & \multicolumn{3}{|l|}{ Plasma } & \multicolumn{3}{|l|}{ Urine } \\
\hline & Mean, mg/L & $\mathrm{CV} \%$ & $\mathrm{n}$ & Mean, mg/L & $\mathrm{CV} \%$ & $\mathrm{n}$ \\
\hline \multicolumn{7}{|c|}{ Intra-assay } \\
\hline Low & 3.06 & 6.75 & 10 & 0.56 & 15.59 & 11 \\
\hline High & 10.11 & 3.86 & 10 & 4.84 & 12.15 & 11 \\
\hline \multicolumn{7}{|c|}{ Inter-assay } \\
\hline Low & 5.02 & 10.22 & 29 & 0.44 & 16.15 & 23 \\
\hline High & 11.03 & 11.13 & 29 & 5.63 & 10.53 & 26 \\
\hline
\end{tabular}

Table 2 Linearity results for Gb3 quantification (dilution series).

\begin{tabular}{llll}
\hline Dilution series & Plasma & Urine low & Urine high \\
\hline Mean value $\pm \mathrm{SD}, \mathrm{mg} / \mathrm{L}$ & $10.98 \pm 0.38$ & $3.14 \pm 0.18$ & $6.81 \pm 0.10$ \\
$\mathrm{CV} \%$ & 3.50 & 5.61 & 1.48 \\
Recovery $\pm \mathrm{SD}, \%$ & $100.55 \pm 3.69$ & $100.14 \pm 3.77$ & $100.04 \pm 1.78$ \\
Recovery max, \% & 104.8 & 107.8 & 102.7 \\
Recovery min, \% & 92.3 & 94.8 & 96.2 \\
Linear range, mg/L & $0.54-10.86$ & $0.07-3.28$ & $0.17-6.72$ \\
Linear regression & $\mathrm{y}=1.0362 \mathrm{x}-0.1598$ & $\mathrm{y}=1.0030 \mathrm{x}-0.0122$ & $\mathrm{y}=1.0028 \mathrm{x}+0.0046$ \\
Regression coefficient, $\mathrm{r}^{2}$ & 0.9957 & 0.9993 & 0.9997 \\
\hline
\end{tabular}

Table 3 Stability data following storage at $-20^{\circ} \mathrm{C}$ (each value is the mean of six analyses).

\begin{tabular}{|c|c|c|c|c|c|}
\hline \multirow[t]{2}{*}{ Pool } & \multirow[t]{2}{*}{ Storage time, year } & \multicolumn{2}{|l|}{ Plasma } & \multicolumn{2}{|l|}{ Urine } \\
\hline & & Mean, mg/L & Deviation, $\%$ & Mean, mg/L & Deviation, $\%$ \\
\hline Low & $1 / 2$ & 4.82 & -2.2 & 0.57 & -10.5 \\
\hline High & $1 / 2$ & 9.92 & +2.1 & 5.91 & +2.7 \\
\hline Low & 1 & 4.96 & +3.2 & 0.47 & -21.1 \\
\hline High & 1 & 10.97 & -1.35 & 5.49 & +5.7 \\
\hline
\end{tabular}



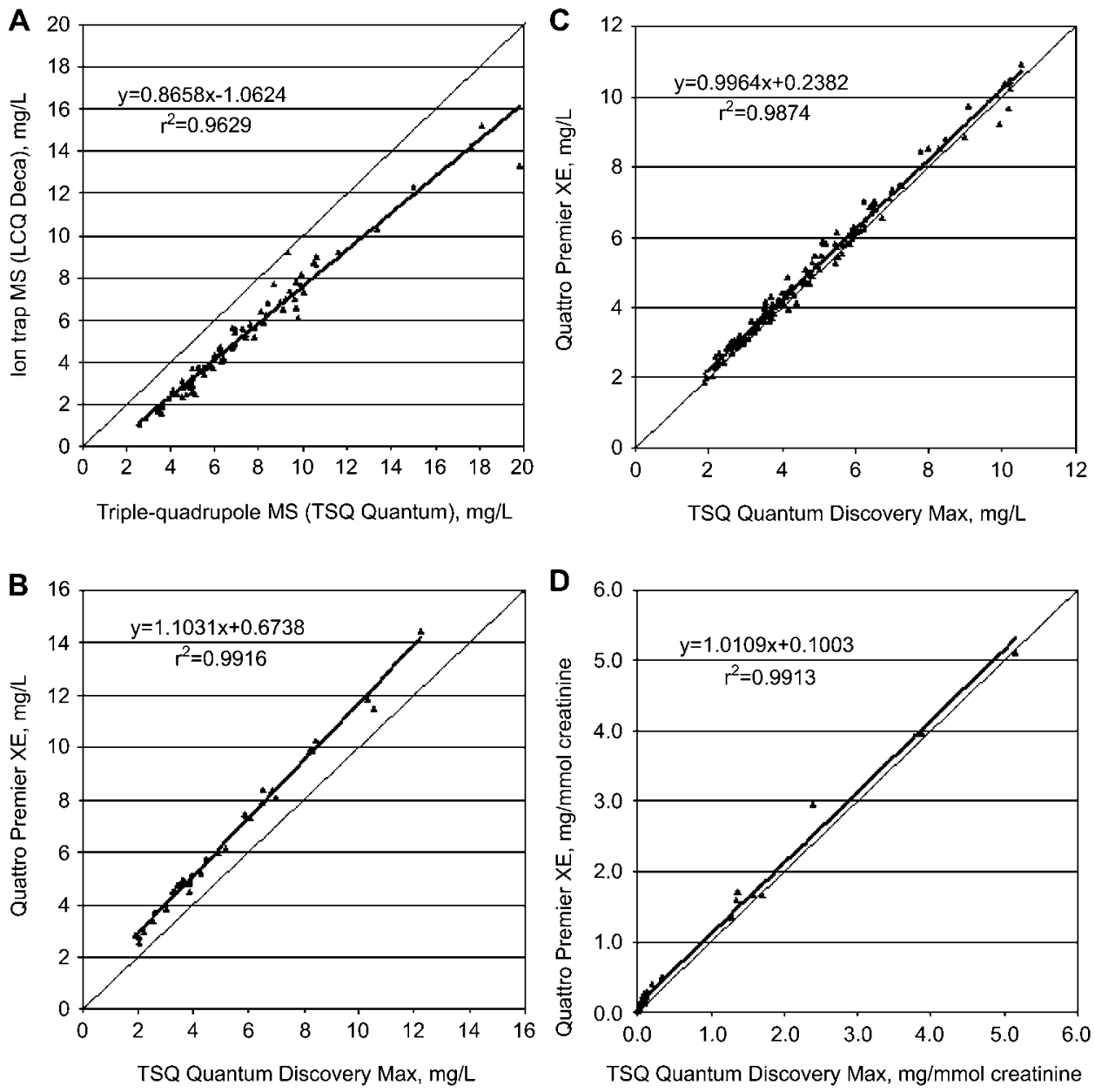

Figure 3 Instrument comparison.

Gb3 was extracted from EDTA plasma or urine of patients with confirmed and suspected Fabry disease, and the purified samples were analyzed with different mass spectrometers. (A) Comparison of two mass spectrometer types $(\mathrm{n}=89)$ : a triple-quadrupole mass spectrometer (TSQ Quantum Discovery Max, Thermo Scientific) and a quadrupole ion trap (LCQ Deca XP, Thermo Scientific). Both instruments were equipped with Surveyor autosampler and HPLC pump. (B-D) Comparison of two different triple quadrupole mass spectrometers: TSQ Quantum Discovery Max (Thermo Scientific) equipped with a Surveyor autosampler and HPLC pump, and Quattro Premier XE (Waters) equipped with Acquity autosampler and UPLC pump. (B) shows the obtained correlation using identical collision voltage (62 V) and similar quadrupole resolution $(n=33)$. (C) (EDTA-plasma, $n=93$ ) and (D) (urine, $n=35)$ show the obtained correlations after careful adjustment of instrument parameters.

observed even between the two triple quadrupoles (see Figure 3B). The values obtained on the Quattro Premier were significantly higher, although the correlation was excellent with an $\mathrm{r}^{2}>0.99$.

At first glance, no difference would be expected using the same type of instrument. However, heterogeneity of Gb3 may influence quantification results. Since no obvious difference was observed in the full scan survey spectra (see Figure 2), we examined whether different isoform profiles appear in the MRM modus. The relative MRM abundancies of human Gb3 isoforms in plasma and urine from patients with Fabry disease, before and after spiking with porcine Gb3 standard, are shown in Figure 4.

In addition to the obvious differences between plasma and urine, and also upon spiking, small but discernable deviations in the isoform profiles were observed with the two triple quadrupoles. We suspected that this effect might be related to different fragmentation efficiencies and/or resolution settings. This assumption and the influence of these parameters on the isoform profile were verified by recording Gb3 MRM spectra at different collision offsets and quadrupole resolution settings (see Figure 5).

After this finding, tuning conditions were adapted such that almost identical isoform abundancies were obtained on either instrument. It turned out that the appropriate collision energy was $68 \mathrm{eV}$ using the Quattro Premier, in contrast to $62 \mathrm{eV}$ with the TSQ Quantum. With these new settings, correlation was now appropriate with negligible quantitative deviations (see Figure 3C and D).

\section{Gb3 levels in Fabry patients and healthy controls}

The successful evaluation of the method qualifies its routine application in a clinical setting. However, the differences 

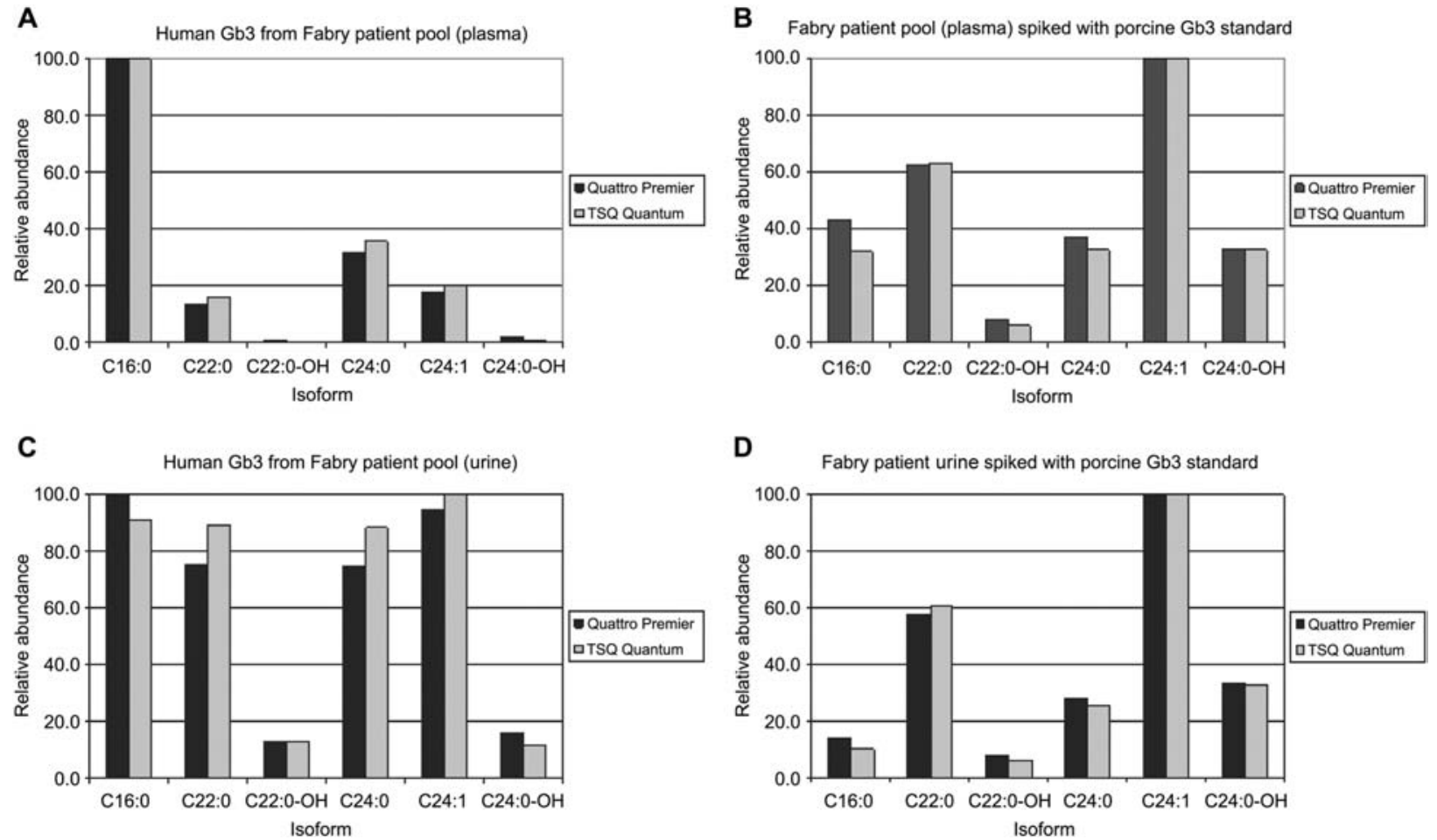

Figure 4 Relative MRM abundancies of Gb3 isoforms in plasma and urine of Fabry patients before and after spiking with porcine Gb3 standard.

The obtained average isoform abundancies ( $\mathrm{m} / \mathrm{z}$ values) were normalized by setting the highest isoform to $100 \%$. The bar graphs on the left show the intensity distribution in plasma (A) and urine (C) of Fabry patients. The bar graphs on the right depict the situation after adding the porcine Gb3 spike (B: plasma, spike $19.2 \mathrm{mg} / \mathrm{L}$; D: urine, spike $7.2 \mathrm{mg} / \mathrm{L}$ ). For comparison, the results obtained with two different mass spectrometers are shown. It is readily apparent that the lower isoforms ( $\mathrm{m} / \mathrm{z} 1046)$ are underrepresented in the TSQ Quantum (Thermo Scientific) compared to the Quattro Premier XE (Waters). Moreover, plasma and urine show considerably different isoform profiles.

observed on various MS platforms support the necessity for determining method-specific reference ranges. Gb3 concentrations in the patients with Fabry disease and controls are summarized in Table 4. All ranges are shown as the 5th-95th percentile.

Gb3 concentrations in 50 EDTA plasma samples from healthy controls were determined with the new method using the triple quadrupole (TSQ Quantum). The median was $2.8 \mathrm{mg} / \mathrm{L}$, with a range of $1.7-3.6 \mathrm{mg} / \mathrm{L}$ (see Figure 6A). Slightly lower results $(15 \%)$ were obtained with the quadrupole ion trap (LCQ Deca), reflecting exactly the different responses of the two instruments. However, the upper limit of the 95th percentile was almost identical.

As expected, patients with Fabry disease show significantly higher Gb3 plasma concentrations $(\mathrm{p}<0.0001)$. The median for samples from male Fabry patients $(n=34)$ was $6.6 \mathrm{mg} / \mathrm{L}$ with a range of $2.9-13.3 \mathrm{mg} / \mathrm{L}$. The median for samples from female heterozygotes $(\mathrm{n}=58)$ was lower at $4.1 \mathrm{mg} / \mathrm{L}$, with a range of $2.4-5.7 \mathrm{mg} / \mathrm{L}$.

Gb3 concentrations in urine are considerably lower, and values near the limit of quantification are often observed, even in Fabry patients (see Figure 6B). Gb3 values were normalized to creatinine to correct for differences in urinary concentrations. Values were determined with the TSQ Quantum. The median in healthy controls $(n=89)$ was 0.8 $\mu \mathrm{g} / \mathrm{mmol}$ creatinine, with a range of $0.4-3.0 \mu \mathrm{g} / \mathrm{mmol}$ creatinine. Gb3 concentrations were also significantly increased $(\mathrm{p}<0.0001)$ in the urine of female $(\mathrm{n}=57)$ and male $(\mathrm{n}=22)$ Fabry patients. The median Gb3 concentrations was $28 \mu \mathrm{g} / \mathrm{mmol}$ creatinine in women (range 1-96), and 218 $\mu \mathrm{g} / \mathrm{mmol}$ creatinine in men (range 3-584).

Since ERT is expected to lower Gb3 concentrations in plasma and urine, we also analyzed an independent group of patients who were undergoing ERT for at least 6 months at the time of blood or urine collection. There was a trend for lower plasma and urine concentrations of Gb3 (see Table 4). However, this difference did not reach statistical significance. If $\mathrm{Gb} 3$ concentrations were analyzed in individual patients before and after ERT, median plasma Gb3 decreased from 9.4 to $5.3 \mathrm{mg} / \mathrm{L}$ in men $(\mathrm{p}=0.005)$, but was little changed in women ( 3.9 and $3.8 \mathrm{mg} / \mathrm{L}, \mathrm{p}=0.557)$. Median urinary Gb3 decreased from 285 to $165 \mu \mathrm{g} / \mathrm{mmol}$ creatinine in men, albeit with little significance $(\mathrm{p}=0.524)$, and from 30 to $9 \mu \mathrm{g} / \mathrm{mmol}$ creatinine in women $(\mathrm{p}=0.056)$.

Several reports suggest a correlation between genotype and Gb3 concentrations (12, 13, 34). Therefore, we sorted the patients into three groups: non-sense mutations, missense mutations, and deletions/insertions and splice site defects. Due to the small number of patients, there were no significant differences, even though patients with missense mutations tended to have lower plasma and urinary Gb3 concentrations. All men with Gb3 plasma concentrations 

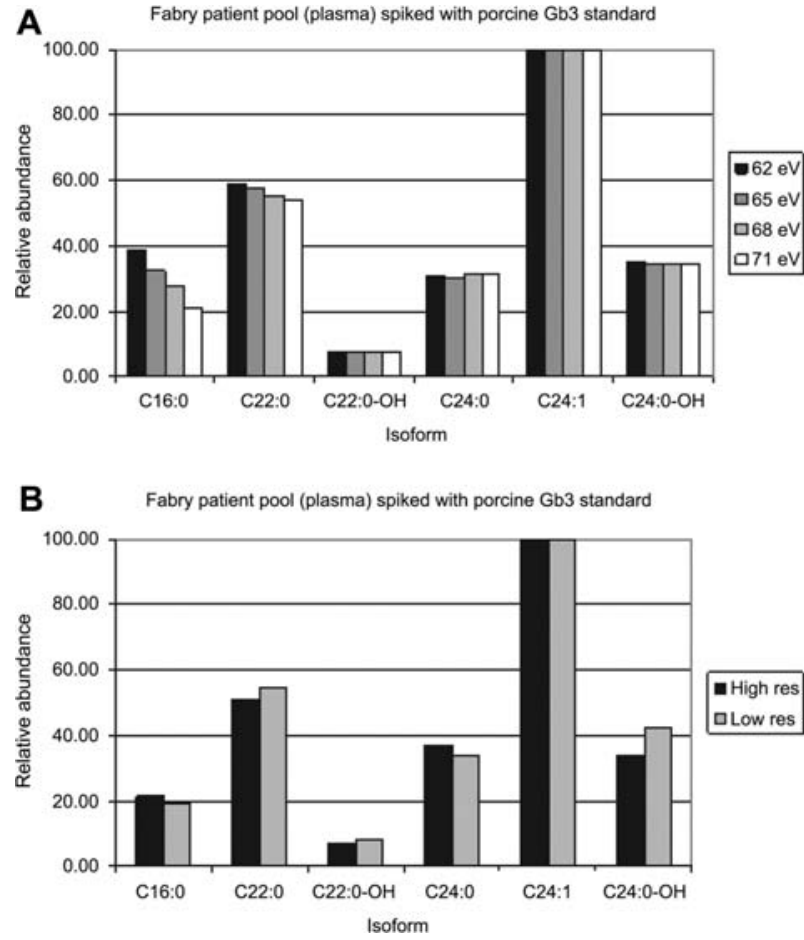

Figure $5 \mathrm{~Gb} 3$ isoform profiles.

Gb3 isoform profiles obtained with different collision energies (A) and quadrupole resolution settings (B) using a triple quadrupole (Quattro Premier XE, Waters). The obtained average isoform abundancies $(\mathrm{m} / \mathrm{z}$ values) were normalized by setting the highest isoform to $100 \%$.

within the reference range had missense mutations, which is consistent with previous reports (34). However, from eight patients (four male, four female) with the cardiac variants $\mathrm{R} 112 \mathrm{H}$ or N215S, two women and one male presented with moderately, but clearly increased plasma concentrations up to $5 \mathrm{mg} / \mathrm{L}$, albeit normal urine Gb3.

\section{Discussion}

\section{Methodic considerations and method performance}

Heterogeneity and the amphiphilic nature of Gb3 dominates considerations for establishment of a routine test. In order to avoid surface adsorption, tailing and memory effects on the column, we chose offline sample preparation using sequential liquid extraction and SPE instead of online LC-MS. Protein precipitation and Gb3 extraction with acetone: methanol (1:1/v:v) gave good results with EDTA plasma. Lipid extraction with chloroform:methanol (2:1/v:v) was used for urine. In contrast to other protocols $(30,32)$, analytes were enriched using a silica stationary phase as a second purification step. Due to an initial liquid extraction and subsequent redissolution in pure chloroform, proteins and the majority of polar contaminants and salts are eliminated before samples are applied to the normal phase column. Subsequently, lipids, such as triglycerides and cholesterol esters are washed off the column with chloroform, whereas amphiphilic analytes with polar moieties are trapped on the silica and can be eluted with solvents of higher polarity. Acetone:methanol 4:1 (v:v) was found to be suitable for elution of Gb3.

Since calibration with pure standards is not possible due to strong matrix suppression effects, and matrix-matched human Gb3 standards are not available, we decided in favor of standard addition instead. In addition, differences during sample preparation and fluctuations during ESI detection are compensated by addition of a suited ISTD with a non-natural fatty acid (C17), synthesized from natural Gb3 by reacylation of deacylated Gb3 with heptadecanoic acid (28). With respect to the ISTD, it is important to mention that FIA has another advantage compared to online LC-MS: compensation for ionization fluctuations and matrix suppression effects requires simultaneous elution of analyte and ISTD into the MS. This is easily achieved by FIA, whereas chromatographic separation of different Gb3 isoforms, including the ISTD, is difficult to avoid during LC-MS.

The variability of isoform abundancies is another issue to be addressed. The only available source for calibration standards, Gb3 from porcine erythrocytes, contains primarily isoforms with comparably long fatty acid chains (mainly C22, C24, see Figure 2). In contrast, isoforms with short fatty acids are most abundant in blood plasma, especially C16:0 (m/z 1046, sodiated precursor). Longer fatty acids are usually found in urine (30). It should be noted that Nelson et al. observed strong variations of isoform profiles with LC-MS, requiring thorough rinsing (32). Since absorption by capillary walls and in the injector is critical, even during FIA, the inlet was washed after each sample by injection of methanol blanks using short 1.5 min runs.

Table 4 Gb3 concentrations of the patient groups with and without enzyme replacement therapy (ERT); range $=5$ th -95 th percentile .

\begin{tabular}{|c|c|c|c|c|c|c|c|c|}
\hline & \multicolumn{4}{|c|}{ Plasma Gb3, mg/L } & \multicolumn{4}{|c|}{ Urine $\mathrm{Gb} 3, \mu \mathrm{g} / \mathrm{mmol}$ creatinine } \\
\hline & $\mathrm{n}$ & Mean & Median & Range & $\mathrm{n}$ & Mean & Median & Range \\
\hline Controls & 50 & 2.8 & 2.8 & $1.7-3.6$ & 89 & 1 & 0.8 & $0.4-3$ \\
\hline \multicolumn{9}{|l|}{ Fabry male } \\
\hline Without ERT & 34 & 7.3 & 6.6 & $2.9-13.3$ & 22 & 221 & 218 & $3-584$ \\
\hline ERT & 53 & 6.2 & 5.9 & $2.8-11.2$ & 49 & 261 & 52 & $2-907$ \\
\hline \multicolumn{9}{|l|}{ Fabry female } \\
\hline Without ERT & 58 & 4.1 & 4.1 & $2.4-5.7$ & 57 & 33 & 28 & $1-96$ \\
\hline ERT & 64 & 4.2 & 4.1 & $2.7-5.9$ & 60 & 22 & 12 & $3-50$ \\
\hline
\end{tabular}



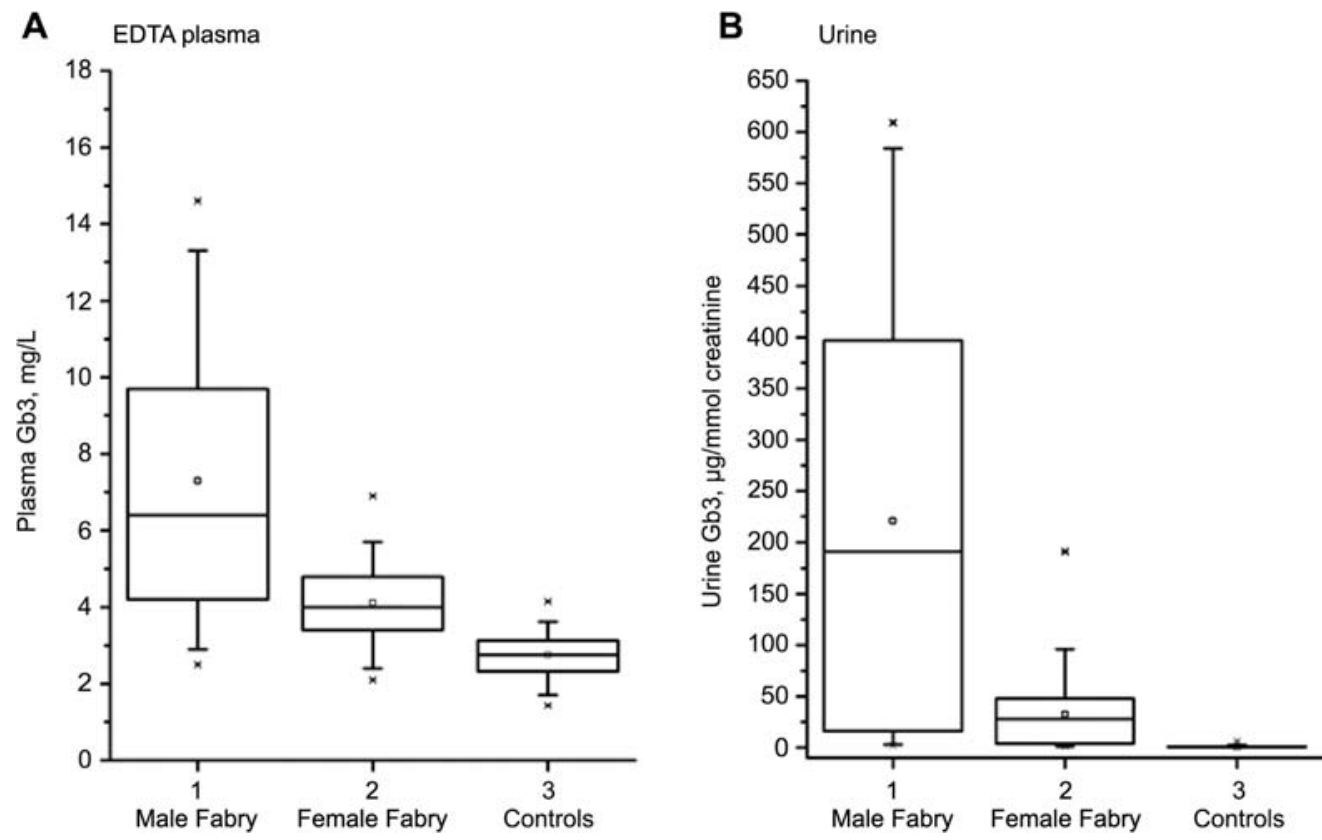

Figure 6 Comparison of plasma and urine Gb3 concentrations in Fabry patients without ERT and healthy controls. (A) Gb3 concentrations in EDTA plasma samples from 34 male Fabry patients, 58 female Fabry patients and 50 healthy controls were determined with the described method using the TSQ Quantum triple quadrupole. Both groups were found to be significantly different from the control group with $\mathrm{p}<0.0001$. Median Gb3 concentrations in $\mathrm{mg} / \mathrm{L}$ were: male Fabry patients 6.6 (mean 7.3), female Fabry patients 4.1 (mean 4.1), and controls 2.8 (mean 2.8). (B) Gb3 concentrations in urine samples from 22 male Fabry patients, 57 female Fabry patients and 89 healthy controls were determined with the described method using the TSQ Quantum triple quadrupole. Both groups were found to be significantly different from the control group with $\mathrm{p}<0.0001$. Median Gb3 levels in $\mu \mathrm{g} / \mathrm{mmol}$ creatinine were: male Fabry patients 218 (mean 221), female Fabry patients 28 (mean 33), and controls 0.8 (mean 1). The square represents the mean average and the line within the box represents the median. The box represents the 25 th-75th percentile, and the whiskers represent the 5th-95th percentile (see text for range numbers). The crosses represent the 1st-99th percentile, and the dash gives the maximum and minimum values, respectively.

In order to cover the heterogeneous isoform profiles of all materials, total $\mathrm{Gb} 3$ was calculated by summing the most abundant isoforms present in plasma, urine and porcine $\mathrm{Gb3}$. However, a certain intrinsic bias cannot be completely avoided since the number of parallel MRM transitions is limited, and monitoring a high number of isoforms leads to poor duty cycles and low intensities. Monitoring of six isoforms plus ISTD was a good compromise between accuracy and signal intensity, and produced reproducible results. The method covers the concentration range for monitoring $\mathrm{Gb} 3$ in plasma and urine, and allows for accurate and robust long-term monitoring of Fabry patients.

\section{Influence of isoform profiles and instrument comparison}

The observed deviations between different mass spectrometers are related to the fact that mass-dependent sensitivity is instrument-dependent and can influence the relative signal height of Gb3 isoforms. Due to different abundancies of isoforms in standards and samples, any mass-dependent sensitivity difference would necessarily result in systematic deviations upon calibration. For instance, CID fragmentation in ion traps occurs via multiple low-energy collisions, whereas single high-energy collisions are the dominant fragmentation pathway in collision-cells of triple quadrupoles. Moreover, the limited linear range and ion capacity of ion traps is known to affect quantitation performance. Hence, the observed instrumental deviation between ion trap and triple quadrupole may be expected. However, the good correlation indicates that reliable and reproducible results are obtained on either instrument.

At first glance, no deviation would be expected between two triple quadrupoles. However, there are several instrument differences and parameter settings that might lead to changes in the isoform profiles obtained. For example, ion desolvation in the TSQ Quantum is achieved with use of a heated capillary, whereas the Quattro Premier utilizes a heated nitrogen counter flow. Differences in collision efficiency may also lead to varying detection efficiencies upon MRM of Gb3 isoforms, even at identical collision energies. Indeed, experiments showed that collision energy and quadrupole resolution had the greatest influence on isoform distribution, and remarkably different collision energies were necessary in order to obtain comparable isoform profiles and consistent quantitative results with the two instruments. The influence of the quadrupole resolution can be explained by crosstalk from neighbored isoform isotopes, especially if low resolution settings are used. Most likely, the effect of collision energy reflects the influence of the fatty acid chain length on activation energy, and therefore fragmentation efficiency.

It has to be noted that due to the lack of certified reference standards and methods, there is currently no statement possible about the accuracy of either method. In principal, dif- 
ferent methods can be used successfully even without exact matching of the results, provided appropriate reference ranges are taken into account. However, universal reference ranges are highly preferable, requiring careful adjustment of instrumental conditions to ensure equal results.

\section{Reference ranges}

Male and female Fabry patients have significantly higher Gb3 plasma concentrations compared to controls. Although low Gb3 concentrations do not necessarily exclude the existence of Fabry disease, especially in women who generally show lower Gb3 concentrations, high Gb3 values above $4.0 \mathrm{mg} / \mathrm{L}$ strongly support the diagnosis of Fabry (3rd-97th percentile TSQ, 5th-95th percentile LCQ). Consequently, the cut-off for the routine assay was set to $4.0 \mathrm{mg} / \mathrm{L}$. At this cutoff threshold, specificity was $98 \%$, with a sensitivity of $79 \%$ for male Fabry patients and a sensitivity of $54 \%$ for female Fabry patients.

In urine from healthy controls, very low Gb3 concentrations were measured that were essentially equivalent to the background of the assay. Consequently, urinary Gb3 $>10 \mu \mathrm{g} / \mathrm{mmol}$ creatinine gives strong evidence for Fabry disease. At this cut-off, specificity was $100 \%$, with a sensitivity of $77 \%$ for male Fabry patients and a sensitivity of $65 \%$ for female Fabry patients.

Significantly increased urine Gb3 concentrations in Fabry patients were frequently observed, occasionally above $1000 \mu \mathrm{g} / \mathrm{mmol}$ creatinine. However, many Fabry patients have very low urinary Gb3 concentrations, and normal urine Gb3 does not exclude the possibility of Fabry, similar to the situation in plasma. In addition, increased urine concentrations do not necessarily coincide with increased plasma values. A correlation with the type of mutation was suggested to explain this observation (12).

However, it remains still unclear as to what extent genetic variations are important for the abundance of Gb3 in plasma or urine of Fabry patients. We found only weak correlation with the type of mutation, except that patients with low Gb3 usually have missense mutations. In contrast, we also observed patients with cardiac variants and increased Gb3 plasma concentrations. These observations indicate that a simple classification is probably not appropriate, and individual mutations need to be treated separately with respect to the effect on enzyme structure and Fabry outcome. Further investigations on this topic will be performed.

\section{Conflict of interest statement}

Authors' conflict of interest disclosure: The authors stated that there are no conflicts of interest regarding the publication of this article. Research funding played no role in the study design; in the collection, analysis, and interpretation of data; in the writing of the report; or in the decision to submit the report for publication.

Research funding: Financial support of Shire Human Genetics Therapies is gratefully acknowledged.

Employment or leadership: None declared.

Honorarium: None declared.

\section{References}

1. Brady RO. Enzymatic defect in Fabry's disease. Ceramidetrihexosidase deficiency. N Engl J Med 1967;276:1163-7.

2. Fabry H. An historical overview of Fabry disease. J Inherit Metab Dis 2001;24(Suppl 2):3-7.

3. Clarke JT. Narrative review: Fabry disease. Ann Intern Med 2007;146:425-33.

4. Eng CM, Desnick RJ. Molecular basis of Fabry disease: mutations and polymorphisms in the human alpha-galactosidase A gene. Hum Mutat 1994;3:103-11.

5. Garman SC, Garboczi DN. The molecular defect leading to Fabry disease: structure of human alpha-galactosidase. J Mol Biol 2004;337:319-35.

6. Desnick RJ, Brady R, Barranger J, Collins AJ, Germain DP, Goldman M, et al. Fabry disease, an under-recognized multisystemic disorder: expert recommendations for diagnosis, management, and enzyme replacement therapy. Ann Intern Med 2003; 138:338-46.

7. Hauser AC, Lorenz M, Sunder-Plassmann G. The expanding clinical spectrum of Anderson-Fabry disease: a challenge to diagnosis in the novel era of enzyme replacement therapy. $\mathbf{J}$ Intern Med 2004;255:629-36.

8. MacDermot KD, Holmes A, Miners AH. Anderson-Fabry disease: clinical manifestations and impact of disease in a cohort of 98 hemizygous males. J Med Genet 2001;38:750-60.

9. MacDermot KD, Holmes A, Miners AH. Anderson-Fabry disease: clinical manifestations and impact of disease in a cohort of 60 obligate carrier females. J Med Genet 2001;38:769-75.

10. Rolfs A, Böttcher T, Zschiesche M, Morris P, Winchester B, Bauer P, et al. Prevalence of Fabry disease in patients with cryptogenic stroke: a prospective study. Lancet 2005;366: 1794-6.

11. Vedder AC, Linthorst GE, van Breemen MJ, Groener JE, Bemelman FJ, Strylond A, et al. The Dutch Fabry cohort: diversity of clinical manifestations and Gb3 levels. J Inherit Metab Dis 2007;30:68-78.

12. Auray-Blais C, Cyr D, Ntwari A, West ML, Cox-Brinkman J, Bichet DG, et al. Urinary globotriaosylceramide excretion correlates with the genotype in children and adults with Fabry disease. Mol Genet Metab 2008;93:331-40.

13. Kitagawa $\mathrm{T}$, Ishige $\mathrm{N}$, Suzuki K, Owada M, Ohashi T, Kobayashi M, et al. Non-invasive screening method for Fabry disease by measuring globotriaosylceramide in whole urine samples using tandem mass spectrometry. Mol Genet Metab 2005;85:196-202.

14. Beck M. New therapeutic options for lysosomal storage disorders: enzyme replacement, small molecules and gene therapy. Hum Genet 2007;121:1-22.

15. Vance DE, Sweeley CC. Quantitative determination of the neutral glycosyl ceramides in human blood. J Lipid Res 1967; 8:621-30.

16. Gross SK, McCluer RH. High-performance liquid chromatographic analysis of neutral glycosphingolipids as their per-Obenzoyl derivatives. Anal Biochem 1980;102:429-33.

17. Hazumi I, Nishizawa M, Ariga T, Miyatake T. Biochemical and clinical analysis of accumulated glycolipids in symptomatic heterozygotes of angiokeratoma corporis diffusum (Fabry's disease) in comparison with hemizygotes. J Lipid Res 1990; 31:335-40.

18. Kniep B, Mühlradt PF. Immunochemical detection of glycosphingolipids on thin-layer chromatograms. Anal Biochem 1990;188:5-8. 
19. Groener JE, Poorthuis BJ, Kuiper S, Helmond MT, Hollak CE, Aerts JM. HPLC for simultaneous quantification of total ceramide, glucosylceramide, and ceramide trihexoside concentrations in plasma. Clin Chem 2007;53:742-7.

20. Ullmann MD, McCluer RH. Quantitative microanalysis of perbenzoylated neutral glycosphingolipids by high-performance liquid chromatography with detection at $230 \mathrm{~nm}$. J Lipid Res 1978;19:910-3.

21. Lee WM, Westrick MA, Macher BA. High-performance liquid chromatography of long-chain neutral glycosphingolipids and gangliosides. Biochim Biophys Acta 1982;7:498-504.

22. Strasberg PM, Warren I, Skomorowski MA, Lowden JA. HPLC analysis of neutral glycolipids: an aid in the diagnosis of lysosomal storage disease. Clin Chim Acta 1983;132:29-41.

23. Roy S, Gaudin K, Germain DP, Baillet A, Prognon P, Chaminade P. Optimisation of the separation of four major neutral glycosphingolipids: application to a rapid and simple detection of urinary globotriaosylceramide in Fabry disease. J Chromatogr B 2004;805:331-7.

24. Berná L, Asfaw B, Conzelmann E, Černý B, Ledvinova J. Determination of urinary sulfatides and other lipids by combination of reversed-phase and thin-layer chromatographies. Anal Biochem 1999;269:304-11.

25. Zeidner KM, Desnick RJ, Ioannou YA. Quantitative determination of globotriaosylceramide by immunodetection of glycolipid-bound recombinant verotoxin B subunit. Anal Biochem 1999;267:104-13.

26. Nakanishi T, Funahashi S, Funai T, Hashimoto T, Shimizu A. Chemical diagnosis of Fabry's disease by fluorometric assay and fast atom bombardment/mass spectrometry. Ann Clin Biochem 1991;28:368-72.

27. Touboul D, Roy S, Germain DP, Baillet A, Brion F, Prognon $\mathrm{P}$, et al. Fast fingerprinting by MALDI-TOF mass spectrometry of urinary sediment glycosphingolipids in Fabry disease. Anal Bioanal Chem 2005;382:1209-16.

28. Mills K, Johnson A, Winchester B. Synthesis of novel internal standards for the quantitative determination of plasma ceramide trihexoside in Fabry disease by tandem mass spectrometry. FEBS Lett 2002;515:171-6.

29. Boscaro F, Pieraccini P, la Marca G, Bartolucci G, Luceri L, Luceri F, et al. Rapid quantitation of globotriaosylceramide in human plasma and urine: a potential application for monitoring enzyme replacement therapy in Anderson-Fabry disease. Rapid Commun Mass Spectrom 2002;16:1507-14.
30. Fauler G, Rechberger GN, Devrnja D, Erwa W, Plecko B, Kotanko P, et al. Rapid determination of urinary globotriaosylceramide isoform profiles by electrospray ionization mass spectrometry using stearoyl-d35-globotriaosylceramide as internal standard. Rapid Commun Mass Spectrom 2005;19:1499-506.

31. Whitfield PD, Calvin J, Hogg S, O'Driscoll E, Halsall D, Burling $\mathrm{K}$, et al. Monitoring enzyme replacement therapy in Fabry disease - role of urine globotriaosylceramide. J Inherit Metab Dis 2005;28:21-33.

32. Nelson BC, Roddya T, Araghi S, Wilkens D, Thomas JT, Zhang $\mathrm{K}$, et al. Globotriaosylceramide isoform profiles in human plasma by liquid chromatography-tandem mass spectrometry. J Chromatogr B 2004;805:127-34.

33. Mills K, Vellodi A, Morris P, Cooper D, Morris M, Young E, et al. Monitoring the clinical and biochemical response to enzyme replacement therapy in three children with Fabry disease. Eur J Pediatr 2004;163:595-603.

34. Mills K, Morris P, Lee P, Vellodi A, Waldek S, Young E, et al. Measurement of urinary $\mathrm{CDH}$ and $\mathrm{CTH}$ by tandem mass spectrometry in patients hemizygous and heterozygous for Fabry disease. J Inherit Metab Dis 2005;28:35-48.

35. Nelson C, Sung CC, Araghi S, Wilkens D, Zhang K, Thomas $\mathrm{JJ}$, et al. Liquid chromatography-tandem mass spectrometry quantification of globotriaosylceramide in plasma for long-term monitoring of Fabry patients treated with enzyme replacement therapy. Clin Chem 2005;51:237-40.

36. Delobel A, Roy S, Touboul D, Gaudin K, Germain DP, Baillet A, et al. Atmospheric pressure photoionization coupled to porous graphitic carbon liquid chromatography for the analysis of globotriaosylceramides. Application to Fabry disease. J Mass Spectrom 2006;41:50-8.

37. Mehta A, Ricci R, Widmer U, Dehout F, Garcia de Lorenzo A, Kampmann C, et al. Fabry disease defined: baseline clinical manifestations of 366 patients in the Fabry Outcome Survey. Eur J Clin Invest 2004;34:236-42.

38. Deegan PB, Baehner AF, Barba Romero MÁ, Hughes DA, Kampmann C, Beck M, et al. Natural history of Fabry disease in females in the Fabry Outcome Survey. J Med Genet 2006; 43:347-52.

39. Ramaswami U, Whybra C, Parini R, Pintos-Morell G, Mehta A, Sunder-Plassmann G, et al. Clinical manifestations of Fabry disease in children: data from the Fabry Outcome Survey. Acta Paediatr 2006;95:86-92. 